\title{
Proceso de objetivación del concepto de fracción cuando los estudiantes resuelven problemas ${ }^{1}$
}

\section{Objectification process fraction concept when students solve problems}

\section{Objetivação conceito de fração processo em que os alunos resolvem problemas}

Recibido: mayo de 2013

Aceptado: agosto de 2013
Maria Andrea Cardona Mesa ${ }^{2}$

Hellen Dadiana Cuesta Romaña ${ }^{3}$

Jhoan Esteban López Román ${ }^{4}$

Leidy Maritza Toro Garcia ${ }^{5}$

\section{Resumen}

La investigación que aquí se presenta responde a la necesidad de hallar el proceso de objetivación del concepto de fracción cuando los estudiantes resuelven problemas, necesidad que nace del estudio de un caso en el cual se identifican dificultades para la resolución de problemas que involucran fracciones. Con un enfoque sociocultural y usando una metodología cualitativa de estudio de casos los investigadores analizan los procesos en los cuales el caso muestra una dotación de sentido de la fracción como parte todo, como relación multiplicativa y como medida en medio de un desencadenamiento de actividad. Del análisis se determinan tres categorías que se consolidan como el proceso de objetivación y que responden al objeto de la investigación.

Palabras clave: Procesos; objetivación; matemáticas escolares; estructuras numéricas; números racionales; fracción; actividad; otras nociones de Educación Matemática; resolución de problemas; resolución y estrategias.

\begin{abstract}
\end{abstract}
The research presented here addresses the need to find the process of objectification of the concept of fractions when students solve problems, need born of a case study that identifies difficulty solving problems involving fractions. With a sociocultural approach using a qualitative methodology and case study researchers analyzed the processes in which the case shows a strength of sense of fraction as part all as multiplicative relationship and as a measure amid a triggering activity. The analysis identifies three categories

\footnotetext{
Articulo de Investigación

Universidad de Antioquia, Medellin, Colombia. Contacto: marianca308@gmail.com

Universidad de Antioquia, Medellin, Colombia. Contacto: seres0218@yahoo.es

Universidad de Antioquia, Medellin, Colombia. Contacto: lovo_8905@hotmail.com

Universidad de Antioquia, Medellin, Colombia. Contacto: 1maritza125@gmail.com
} 
that are consolidated as the process of objectification and responders under investigation.

Keywords: Processes, objectification school mathematics, numerical structures, rational numbers, fraction, activity, other notions of Mathematics Education; troubleshooting, resolution and strategies.

\section{Resumo}

A pesquisa aqui apresentada aborda a necessidade de encontrar o processo de objetivação do conceito de frações quando os alunos a resolver problemas, precisa nascer de um estudo de caso que identifica dificuldade para resolver problemas envolvendo frações. Com uma abordagem sociocultural utilizando uma metodologia qualitativa e estudo de caso, os pesquisadores analisaram os processos em que o caso mostra a força do sentimento de fração como parte de tudo como relação multiplicativa e como uma medida em meio a uma atividade de disparo. A análise identifica três categorias que se consolidou como o processo de objetivação e respondedores sob investigação.

Palavras-chave: Processos, matemática escolar objetivação, estruturas numéricas, números racionais, fração, atividade, outras noções de Educação Matemática; solução de problemas, resolução e estratégias.

\section{Problema}

Las observaciones en el aula de clase permitieron identificar que los estudiantes de cuarto grado de básica primaria no encontraban el sentido de fracción, necesario para resolver los problemas que planteaba su profesora. Para los investigadores se hizo prioritario saber los conceptos de la fracción hacia los cuales el estudiante debía apuntar para así dotar de sentido el problema y ejecutar las acciones que lo llevaran a la respuesta. Así el problema apuntó esencialmente a la dotación de sentido de la fracción; pero aún no eran claras las formas por medio de las cuales los estudiantes dotaban de sentido, la respuesta estaría en las acciones ejecutadas por los mismos estudiantes impulsados por unas necesidades y unos motivos que encontraban en las actividades problemáticas, es decir, en la actividad que se desencadenaba para la resolución del problema. En definitiva el problema que iniciaba como una dificultad para resolver problemas con fracciones se había concretado en el problema de hallar el proceso de objetivación del concepto de fracción, en medio del desencadenamiento de actividad matemática correspondiente a la resolución de problemas, lo cual concluye en la pregunta de investigación ¿cuál es el proceso de objetivación del concepto de fracción cuando los estudiantes del grado cuarto de la Institución Educativa La Paz sede John F. Kennedy resuelven problemas?

\section{Marco teórico}

Dada la pregunta de investigación se identifican tres aspectos importantes, los procesos de objetivación, la resolución de problemas y las fracciones, estos son la base del marco teórico.

Con la teoría de la Objetivación, la investigación se enmarca dentro de un enfoque socio-cultural, una teoría de enseñanza y aprendizaje de las matemáticas, desarrollada por Radford quien plantea que el sujeto es quien da lugar al pensamiento, pensamiento que es mediatizado y materializado por medios semióticos cuando el sujeto está en relación con otros o con los objetos, buscando una dotación de sentido de algún objeto conceptual. Esa dotación de sentido está sujeta a unos significados culturales 
que posibilitan un aprendizaje, ese "proceso de elaboración activa de significados, es lo que llamaremos más adelante un proceso de objetivación" (Radford, 2006, p. 113).

Se hace referencia a la resolución de problemas como actividad matemática, por un lado, por las implicaciones que tiene el problema como tal, teniendo en cuenta lo que plantean Abrantes et al. (2002), y Polya (1965) de los cuales se puede extraer que, para que algo sea un problema aparte de que su solución no sea de manera inmediata, debe generar necesidad o interés por encontrar una solución. Por otro lado, porque desde la historia, se ve como la resolución de problemas ha sido un elemento importante para el desarrollo del currículo de las matemáticas (basados en Stanic, 1989)

Finalmente se encogen las fracciones como objeto conceptual por las dificultades evidenciadas por los estudiantes de cuarto grado de la institución educativa la Paz sede John F. Kennedy en cuanto a la construcción del concepto de fracción, construcción que desde un punto de vista educativo involucra detallados procesos cognitivos, sociales y culturales en tanto es el número que representa una relación entre las partes y el todo asumiendo sus diferentes representaciones como la simbolización de ciertas cantidades reunidas en una sola cantidad, elementos que se deben tener muy presentes para evitar concepciones que no se relacionan con las construcciones teóricas ya elaboradas cultural e históricamente por la humanidad. En esta perspectiva Obando (2006) sugiere algunas definiciones para la fracción, enfocándose en diferentes perspectivas y que se tendrán en cuenta en el desarrollo de la presente investigación, estas son: a) La fracción como una relación multiplicativa, b) La fracción como medida y c) La fracción como parte todo.

\section{Metodología}

La investigación se basó en una metodología cualitativa enfocada en el estudio de casos intrínseco, que de acuerdo a unos criterios de selección, el caso quedo conformado por seis participantes: tres estudiantes de cuarto A y tres estudiantes de cuarto B. Para el estudio se emplearon instrumentos de recolección de datos como: Videograbaciones, producciones escritas, cuadros de análisis y observaciones.

La recolección inicio con la ejecución de unos encuentros con los estudiantes seleccionados para el estudio de casos, cada encuentro se componía de dos momentos o sesiones de setenta y cinco minutos que se separaban por un descanso de treinta minutos, para el desarrollo de cada encuentro se diseñaban previamente unos problemas que estimularan intencionalmente el proceso de objetivación y poder obtener los datos necesarios para dar respuesta a la pregunta de investigación; para tal diseño de problemas se tuvo en cuenta los tres sentidos de la fracción (parte todo, relación multiplicativa y medida) las cuales iban determinando el objetivo de cada una de las sesiones de trabajo.

Para analizar las videograbaciones y observaciones realizadas en cada encuentro se construyó un cuadro de análisis teniendo en cuenta elementos claves de la teoría de la objetivación, esto permitió al grupo de investigadores centrar su atención en cuales de estos elementos que implicaban una dotación de sentido del concepto de fracción se daban con mayor frecuencia, determinando así tres categorías que se consolidan como el proceso de objetivación y que responden al objeto de la investigación, estas son: 1) interacción por explicación e interacción por negociación de sentidos, 2) expresiones orales y expresiones escritas y 3 ). Uso de artefactos (denominados en el trabajo de investigación como herramientas)

\section{Análisis}

Por límites en cuanto a la presentación del trabajo se mostrará el análisis de algunas categorías.

Frente a la situación desencadenada por el problema de cocinando con las fracciones de "Necesitamos medir $16 / 4$ de vaso (7,0 onzas) en leche en polvo para realizar los mazapanes (Disponiendo de dos recipientes para medir, un vaso de 7,0 onzas y otro de 3,5 onzas), el Estudiante S: (después de varios 
intentos) platea: “¡Entonces le sacamos la mitad a uno de los pequeños (levantando el vaso de 3,5 onzas), porque esa mitad cuatro veces me da uno de los grandes! (señalando el vaso de 7,0 onzas), (al terminar la expresión espera una validación de sus compañeros y de los investigadores)". Al utilizar el vaso de 3,5 onzas para expresar una medida en términos del vaso de 7,0 onzas el estudiante establece la razón que hay entre las mitades de una unidad de medida y los cuartos de otra unidad (Imagen $\mathrm{E}$ 4.1). Ello quiere decir que hay una conceptualización de fracción como medida, pero el estudiante no se queda allí, establece además que al tener cuatro mitades de 3,5 se completa un vaso de 7,0 y esto corresponde a una conceptualización de fracción como parte todo y como relación multiplicativa ya que está pensando en las n-esimas partes que componen el vaso más grande con respecto al vaso más pequeño y además está proyectando, no explícitamente, que la respuesta al problema de medir 16/4 se dará al reunir las partes que ha venido componiendo con las medidas establecidas, es decir la unión de cuatro veces $4 / 4$ (cuatro vasos enteros de 7,0 onzas) equivalentes a 16/4 de vaso

\section{Imagen 1}

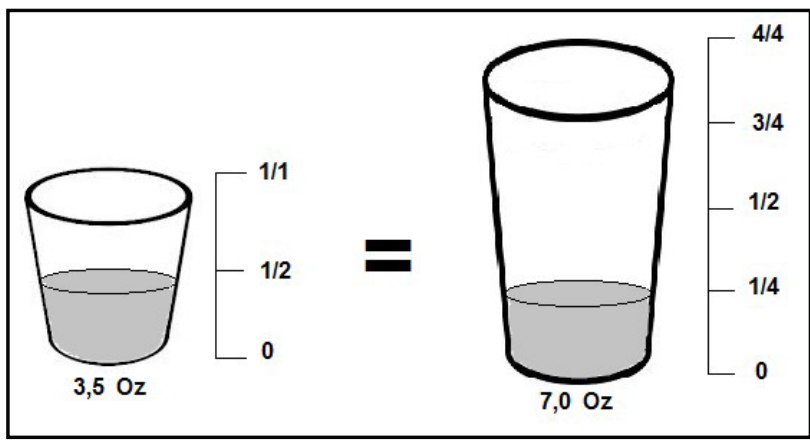

Fuente: Elaboración propia

Con el problema anterior, cuando el investigador plantea que "necesitamos agregar 6/4 de leche condensada del vaso de 7 oz" para la preparación de los mazapanes, el Estudiante $S$ señala en el vaso de 7 onzas, y expresa "más arriba de la mitad", el Estudiante E es un poco más específico dice "si echamos dos de estos (mostrando dos vasos de 3.5 onzas) tenemos 4/4" y el estudiante E plantea "se necesita depositar tres vasos de 3.5 onzas para conseguir los
$6 / 4$ pedidos o si echamos uno de 7 onzas más uno de 3.5 onzas"

Con la herramienta vaso, los estudiantes lograron encontrar la medida pedida para la preparación de los mazapanes, mostrando a través de la actividad manipulativa el paso de lo concreto a lo abstracto de la fracción, al representar con el contenido de los 3 vasos de $3.5 \mathrm{oz}$ los $6 / 4$ del vaso de $7 \mathrm{oz}$ que se necesitaba para agregar a la receta.

\section{Conclusiones}

A partir de la teoría de la objetivación se observa cómo el estudiante que forma parte del estudio del caso es quien da lugar al pensamiento, pensamiento que es mediatizado y materializado por el uso de artefactos como los ingredientes y utensilios para cocinar, además, tienen lugar cuando el estudiante está en relación con los otros y con lo otro a través de interacciones específicas como las explicaciones y la negociación de sentidos. Dando lugar a una dotación de sentido de un objeto conceptual específico, en este caso la fracción.

Teniendo presente que el análisis de evidencias en la actividad matemática denominada "cocinando con las fracciones" permitió identificar las tres categorías de análisis y además considerando que cada uno de ellos estaba fundamentada en los procedimientos de los estudiantes y la teoría de la objetivación, se puede concluir que esas categorías (expresiones orales y escritas, las herramientas y las interacciones por explicación y por negociación) son categorías que para los problemas resueltos describen el proceso de objetivación en la conceptualización de fracción cuando los estudiantes resuelven de problemas.

Considerando que en el análisis de los momentos evidenciados en la actividad hacen referencia constante a la incidencia que tienen unas categorías en otras, es oportuno concluir que las categorías mostrados por el caso no se dan de forma aislada, más bien actúan como referente uno del otro y por lo tanto no es posible determinar el periodo de tiempo exacto en el cual los estudiantes están actuando con una sola de ellas. 


\section{Referencias}

Obando, G. (2006). Pensamiento numérico y sistemas numéricos. Modulo I. Medellín: Artes y Letras Ltda.

Polya, G. (1965). How to solve it. Princenton University Press. México: Ed. Trillas. Traducción hecha por Julián Zugazagoitia

Radford, L. (2006). Elementos de una teoría cultural. Relime,Número Especial , 103-129.
Stanic, G. M. A., \& Kilpatrick, J. (1989). Historical perspectives on problem solving in the mathematics curriculum. In R. I. Charles \& E. A. Silver (Eds.), The teaching and assessing of mathematical problem solving (pp. 1-22). Reston, VA: NCTM e Lawrence Erlbaum. Traducción hecha por Daniel Alexander Arango Restrepo 